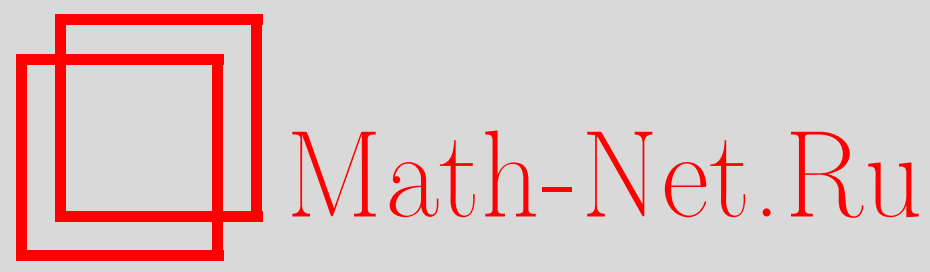

О. Ю. Шведов, Комплексный росток Маслова и асимптотическая формула для канонического распределения Гиббса, Матем. заметки, 1998, том 64, выпуск 4, 622-636

DOI: https://doi.org/10.4213/mzm1438

Использование Общероссийского математического портала Math-Net.Ru подразумевает, что вы прочитали и согласны с пользовательским соглашением http://www.mathnet.ru/rus/agreement

Параметры загрузки:

IP: 52.6 .47 .48

26 апреля 2023 г., 05:31:45

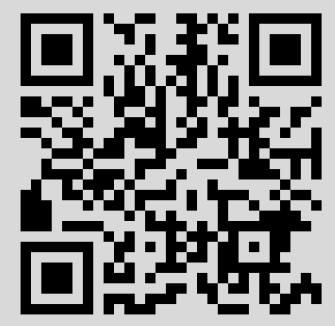




\section{КОМПЛЕКСНЫЙ РОСТОК МАСЛОВА И АСИМПТОТИЧЕСКАЯ ФОРМУЛА ДЛЯ КАНОНИЧЕСКОГО РАСПРЕДЕЛЕНИЯ ГИББСА \\ О. Ю. Шведов}

Рассматривается каноническое распределение Гиббса для системы $N$ классических частиц при следуюших условиях: внешний потенциал порядка 1 , потенциал парного взаимодействия порядка $1 / N$, потенциал тройного взаимодействия порядка $1 / N^{2}$ и т.д. При $N \rightarrow \infty$ строится асимптотика для статистической суммы и свободной энергии. Приводится асимптотическая формула, близкая по норме $L^{1}$ при $N \rightarrow \infty$ к нормированному каноническому распределению. Доказывается, что свойство хаоса вьполнено для частичных распределений и не выполнено для $N$-частичной функции плотности.

Библиограффия: 9 названий.

1. Введение. В равновесной статистической механике для системы $N$ классических частиц рассматривается каноническое распределение Гиббса [1]

$$
\rho_{N}=\frac{1}{Z_{N}} \exp \left(-\frac{H_{N}}{\theta}\right),
$$

зависящее от координат частищ $q_{1}, \ldots, q_{N}$ и импульсов $p_{1}, \ldots, p_{N}$. В формуле $(1) H_{N}-$ функция Гамильтона системы $N$ частиц в $d$-мерном пространстве, $\theta>0$ - температура, $Z_{N}$ - нормировочный множитель (статистическая сумма), определяемый из соотношения

$$
\int \rho_{N} d^{N} p d^{N} q=1 .
$$

В этой работе строится при $N \rightarrow \infty$ асимптотическая формула, аппроксимирующая распределение (1) по норме $L^{1}$, в случае, если функция $H_{N}\left(p_{1}, q_{1}, \ldots, p_{N}, q_{N}\right)$ зависит от $N$ следуюшим образом:

$$
\begin{aligned}
H_{N}\left(p_{1}, q_{1}, \ldots, p_{N}, q_{N}\right)= & \sum_{i=1}^{N}\left(\frac{p_{i}^{2}}{2 m}+g_{1} U_{1}\left(q_{i}\right)\right)+\frac{g_{2}}{N} \sum_{i_{1}, i_{2}=1}^{N} U_{2}\left(q_{i_{1}}, q_{i_{2}}\right)+\cdots \\
& +\frac{g_{p}}{N^{p-1}} \sum_{i_{1}, \ldots, i_{p}=1}^{N} U_{p}\left(q_{i_{1}}, \ldots, q_{i_{p}}\right),
\end{aligned}
$$

Работа выполнена при финансовой поддержке Королевского общества Великобритании. 
где $g_{1}, \ldots, g_{p} \in \mathbb{R}, m \in \mathbb{R}, U_{1}, \ldots, U_{p}$ - гладкие вещественные функции. Функция Гамильтона (2) отвечает следующей физической задаче. Имеется система $N$ частиц массы $m$, находящихся во внешнем поле порядка $O(1)$; потенциал парного взаимодействия имеет порядок $O(1 / N)$, потенциал тройного взаимодействия имеет порядок $O\left(1 / N^{2}\right)$, и т.д.

В работе доказывается, что асимптотическая формула для распределения (1) представляется в виде

$$
\rho_{N}^{\mathrm{as}}\left(p_{1}, q_{1}, \ldots, p_{N}, q_{N}\right)=c_{N}(2 \pi m \theta)^{-N d / 2} \exp \left(-\sum_{i=1}^{N} \frac{p_{i}^{2}}{2 m \theta}\right)\left(\Phi_{N}\left(q_{1}, \ldots, q_{N}\right)\right)^{2},
$$

где

$$
\Phi_{N}\left(q_{1}, \ldots, q_{N}\right)=\sum_{l=0}^{[N / 2]} \frac{1}{(2 N)^{l} l !} \sum_{\substack{i_{1}, \ldots, i_{2 l} \geqslant 1 \\ i_{1} \neq \cdots \neq i_{2 l}}} M\left(q_{i_{1}}, q_{i_{2}}\right) \cdots M\left(q_{i_{2 l-1}}, q_{i_{2 l}}\right) \prod_{j \neq i_{1}, \ldots, i_{2 l}} \varphi\left(q_{j}\right),
$$

$c_{N}$ определяется из условия нормировки

$$
\int \rho_{N}^{\mathrm{as}} d^{N} p d^{N} q=1
$$

функции $M$ и $\varphi$ вводятся ниже.

Отметим, что асимптотики типа (3) часто возникают при применении [2] теории комплексного ростка Маслова [3] к задачам неравновесной статистической механики.

Асимптотическая формула (3) позволяет исследовать проблему хаоса в равновесной статистической механике. Говорят [4], что для $k$-частичного распределения

$$
\rho_{N}^{(k)}\left(p_{1}, q_{1}, \ldots, p_{k}, q_{k}\right)=\int d p_{k+1} d q_{k+1} \cdots d p_{N} d q_{N} \rho_{N}\left(p_{1}, q_{1}, \ldots, p_{N}, q_{N}\right)
$$

выполнено свойство хаоса, если при $N \rightarrow \infty$ это распределение апшроксимируется произведением одночастичных.

В соответствии с результатами работы [2] при $k=$ const, $N \rightarrow \infty k$-частичное распределение (4), отвечающее плотности (3), удовлетворяет свойству хаоса:

$$
\rho_{N}^{(k)}\left(p_{1}, q_{1}, \ldots, p_{k}, q_{k}\right) \rightarrow(2 \pi m \theta)^{-k d / 2} \exp \left(-\frac{p_{1}^{2}+\cdots+p_{k}^{2}}{2 m \theta}\right) \varphi^{2}\left(q_{1}\right) \cdots \varphi^{2}\left(q_{k}\right) .
$$

Формула (5) позволяет вычислять пределы при $N \rightarrow \infty$ средних значений величин типа полной энергии, внутренней энергии, плотности числа частиц, которые выражаются через $k$-частичное распределение при конечном $k$. Из формулы (3) вытекает также, что при $k=N$ свойство хаоса не вьполняется [2].

В работе [5] доказано, что решение задачи Коши для нестационарного уравнения Лиувилля

$$
\left(\frac{\partial}{\partial t}-\sum_{i=1}^{N}\left(\frac{\partial H_{N}}{\partial q_{i}} \frac{\partial}{\partial p_{i}}-\frac{\partial H_{N}}{\partial p_{i}} \frac{\partial}{\partial q_{i}}\right)\right) \rho_{N}\left(t, p_{1}, q_{1}, \ldots, p_{N}, q_{N}\right)=0,
$$

удовлетворяющее при $t=0$ начальному условию (3), сохраняет вид (3) с точностью $O\left(N^{-1 / 2}\right)$ и в остальные моменты времени $t=O(1)$; что распределение (3) совпадает с точностью $O\left(N^{-1 / 2}\right)$ с одним из точных решений (1) уравнения Лиувилля; что формула (3) аппроксимирует решение задачи Коши для уравнения Лиувилля и в том случае, если $t \rightarrow \infty$ при $N \rightarrow \infty$. 
2. Формулировка теоремы. Обозначим через $T$ одномерный тор (окружность). Отождествим точки этого тора с элементами фактормножества $\mathbb{R} / \mathbb{Z}$, которые являются классами эквивалентности $[x]=\{x+n L \mid n \in \mathbb{Z}\}$, где $L$ - вешественное число. Положим $\mathscr{M}=T^{d}=T \times \cdots \times T$. Пусть $U_{l}, l=1, \ldots, p,-$ симметричные гладкие вещественные функции, $U_{l}: \mathscr{M}^{l}=\mathscr{M} \times \cdots \times \mathscr{M} \rightarrow \mathbb{R}$. Рассмотрим функционал $F$, ставящий в соответствие гладкой функции $n: \mathscr{M} \rightarrow \mathbb{R}$ такой, что

$$
\int_{\mathscr{M}} n(x) d x=1
$$

число

$$
F[n(\cdot)]=E[n(\cdot)]+\theta \int d x n(x) \ln n(x),
$$

где

$$
E[n(\cdot)]=g_{1} \int U_{1}(y) n(y) d y+\cdots+g_{p} \int U_{p}\left(y_{1}, \ldots, y_{p}\right) n\left(y_{1}\right) \cdots n\left(y_{p}\right) d y_{1} \cdots d y_{p} .
$$

ЗАмечаниЕ 1. Условие $y_{i} \in \mathscr{M}$ означает, что частицы находятся в кубическом ящике с длиной ребра $L$, причем наложены граничные условия периодичности.

ЗАмЕчАниЕ 2. Функционал $E$ имеет физический смысл внутренней энергии, а $F-$ свободной энергии [1].

Лемма 1. Существует не зависящая от $N$ полохсительная константа $C_{1}$ такая, что при $\left|g_{1} / \theta\right|<C_{1}, \ldots,\left|g_{p} / \theta\right|<C_{1}$ функционал (1) имеет единственный локальный условный әкстремум $n_{0}$ на поверхности (6).

Идея доказательства этой леммы заимствована из работы [6]; доказательство приводится в п. 3 .

Положим

$$
\begin{gathered}
\varphi(x)=\sqrt{n_{0}(x)}, \\
V_{s}\left(x_{1}, \ldots, x_{s}\right)=\frac{1}{s ! \theta} \int d y_{1} \cdots d y_{s}\left(\delta\left(x_{1}-y_{1}\right)-n_{0}\left(y_{1}\right)\right) \cdots\left(\delta\left(x_{s}-y_{s}\right)-n_{0}\left(y_{s}\right)\right) \\
\quad \times \frac{\delta^{s} E}{\delta n\left(y_{1}\right) \cdots \delta n\left(y_{s}\right)}\left[n_{0}(\cdot)\right], \quad s=2, \ldots, p .
\end{gathered}
$$

ЗАмечАниЕ 1. Так как функционал $E$ - полином степени $p$, при $s>p \delta^{s} E=0$, значит, и $V_{s}=0$.

ЗАмЕчАнИЕ 2. Функции $V_{s}$ симметричны по $x_{1}, \ldots, x_{s}$ и удовлетворяют свойству

$$
\int d x_{1} n_{0}\left(x_{1}\right) V_{s}\left(x_{1}, \ldots, x_{s}\right)=0 .
$$

Пусть $A$ - интегральный оператор с ядром,

$$
A(x, y)=\varphi(x) V_{2}(x, y) \varphi(y)
$$

а $M(x, y)$ - ядро оператора $M=-A(1+A)^{-1}(1$ - единичньй оператор).

Положим

$$
f_{0}=-\frac{\theta d}{2} \ln (2 \pi m \theta), \quad Z_{\exp }=\exp \left(-\frac{N\left(F\left[n_{0}(\cdot)\right]+f_{0}\right)}{\theta}\right) .
$$


Tеорема 1. Существует положительная константа $C_{2} \leqslant C_{1}$, не зависящая от $N$, такая, что при $\left|g_{1} / \theta\right|<C_{2}, \ldots,\left|g_{p} / \theta\right|<C_{2}$ справедливы

1) соотношение

$$
\lim _{N \rightarrow \infty} \frac{Z_{N}}{Z_{\exp }}=(\operatorname{det}(1+2 A))^{-1 / 2}
$$

2) асимптотическая формула

$$
\int d p_{1} \cdots d p_{N} d q_{1} \cdots d q_{N}\left|\frac{1}{Z_{\exp }} \exp \left(-\frac{H_{N}}{\theta}\right)-\rho_{N}^{\mathrm{as}}\right| \rightarrow 0, \quad N \rightarrow \infty
$$

здесъ $\rho_{N}^{\text {as }}$ имеет вид $(3)$, а $c_{N}=(\operatorname{det}(1+A))^{-1 / 2}$.

ЗАмечание 1 . Детерминанты $\operatorname{det}(1+A)$ и $\operatorname{det}(1+2 A)$ могут быть определены как

$$
\operatorname{det}(1+\lambda A)=\exp \left(\int_{0}^{\lambda} d \alpha \operatorname{Tr}\left(A(1+\alpha A)^{-1}\right)\right) .
$$

ЗАмЕчАниЕ 2. Величина (11) выражается через детерминант второй вариации свободной энергии

$$
\operatorname{det}(1+2 A)=\frac{1}{\theta} \operatorname{det}\left(\sqrt{n_{0}} \delta^{2} F \sqrt{n_{0}}\right),
$$

где $\sqrt{n_{0}} \delta^{2} F \sqrt{n_{0}}$ - оператор с ядром вида

$$
\sqrt{n_{0}(x)} \frac{\delta^{2} F}{\delta n(x) \delta n(y)} \sqrt{n_{0}(y)}
$$

Асимптотическая формула (3) апроксимирует каноническое распределение с точностью $O\left(N^{-1 / 2}\right)$. Приведем вид асимптотики, аппроксимирующей распределение Гиббса с точностью $O\left(N^{-m / 2}\right)$, где $m$ - любое натуральное число.

Пусть $\varphi \in L^{2}(\mathscr{M}),\|\varphi\|=1$. Обозначим через $\mathscr{F}_{\varphi}^{k}$ пространство симметричных функций $f_{k}\left(x_{1}, \ldots, x_{k}\right)$ из $L^{2}\left(\mathscr{M}^{k}\right)$, удовлетворяющих условию

$$
\int d x_{1} \varphi\left(x_{1}\right) f_{k}\left(x_{1}, \ldots, x_{k}\right)=0
$$

через $\mathscr{F}$ пространство Фока $[7]-$ прямую сумму $\bigoplus_{k=1}^{\infty} \mathscr{F}_{\varphi}^{k}$ со скалярным произведением

$$
(f, g)=\sum_{k=0}^{\infty} \int d x_{1} \cdots d x_{k} f_{k}\left(x_{1}, \ldots, x_{k}\right) g_{k}\left(x_{1}, \ldots, x_{k}\right),
$$

где $f_{k}, g_{k}-k$-е компоненты $f, g$.

Рассмотрим оператор $K_{\varphi, N}: \mathscr{F} \rightarrow L^{2}\left(\mathscr{M}^{N}\right)$,

$$
\left(K_{\varphi, N} f\right)\left(x_{1}, \ldots, x_{N}\right)=\sum_{p=0}^{N} \frac{1}{\sqrt{p ! N^{p}}} \sum_{\substack{1 \leqslant i_{1}, \ldots, i_{p} \leqslant N \\ i_{1} \neq \cdots \neq i_{p}}} f_{p}\left(x_{i_{1}}, \ldots, x_{i_{p}}\right) \prod_{i \neq i_{1}, \ldots, i_{p}} \varphi\left(x_{i}\right)
$$


являющийся частным случаем [8] канонического оператора Маслова для лагранжевого многообразия с комплексным ростком [3].

Определим операторнозначные обобщенные функции [7] $a_{\varphi}^{ \pm}(\xi): \mathscr{F} \varphi \rightarrow \mathscr{F}_{\varphi}$ из соотношений

$$
\begin{gathered}
\left(\int d \xi a_{\varphi}^{-}(\xi) \lambda(\xi) f\right)_{k-1}\left(x_{1}, \ldots, x_{k-1}\right)=\sqrt{k} \int f_{k}\left(\xi, x_{1}, \ldots, x_{k-1}\right) \lambda_{\varphi}(\xi) d \xi \\
\left(\int d \xi a_{\varphi}^{+}(\xi) \lambda(\xi) f\right)_{k}\left(x_{1}, \ldots, x_{k}\right)=\frac{1}{\sqrt{k}} \sum_{j=1}^{k} f_{k-1}\left(x_{1}, \ldots, x_{j-1}, x_{j+1}, \ldots, x_{k}\right) \lambda_{\varphi}\left(x_{j}\right),
\end{gathered}
$$

где

$$
\lambda_{\varphi}(x)=\lambda(x)-\varphi(x) \int d y \lambda(y) \varphi(y) .
$$

Положим

$$
\begin{gathered}
\widehat{\nu}=\int d \xi a_{\varphi}^{+}(\xi) a_{\varphi}^{-}(\xi) \\
\widehat{\varkappa}(y)=\frac{1}{N^{1 / 2}} \varphi(y)\left(a_{\varphi}^{+}(y)+a_{\varphi}^{-}(y)\right)+\frac{1}{N} a_{\varphi}^{+}(y) a_{\varphi}^{-}(y)-\frac{1}{N^{3 / 2}} \widehat{\nu} \varphi(y) a_{\varphi}^{-}(y), \\
B=\frac{N}{2}\left(\int d y_{1} d y_{2} \hat{\varkappa}\left(y_{1}\right) \hat{\varkappa}\left(y_{2}\right) V_{2}\left(y_{1}, y_{2}\right)+\cdots\right. \\
\left.+\int d y_{1} \cdots d y_{p} \widehat{\varkappa}\left(y_{1}\right) \cdots \widehat{\varkappa}\left(y_{p}\right) V_{p}\left(y_{1}, \ldots, y_{p}\right)\right) .
\end{gathered}
$$

Оператор $B$ в пространстве $\mathscr{F} \varphi$ является полиномом по $1 / N^{1 / 2}$ :

$$
B=B_{0}+\frac{1}{N^{1 / 2}} B_{1}+\cdots+\frac{1}{N^{3 p / 2-1}} B_{3 p-2} .
$$

Введем векторы $f_{\alpha}^{s} \in \mathscr{F} \varphi, s=0,1, \ldots, \alpha \in[0,1]$, следующим образом. Пусть $f_{\alpha}^{0}-$ решение уравнения

$$
\frac{d f_{\alpha}^{0}}{d \alpha}=-B_{0} f_{\alpha}^{0}
$$

совпадающеепри $\alpha=0$ с вакуумньм вектором пространства $\Phi$ ока $f_{0}^{0}=(1,0,0, \ldots) \in \mathscr{F} \varphi$. Вектор $f_{\alpha}^{0}$ может быть представлен в виде

$$
f_{\alpha}^{0}=c_{\alpha} \exp \left(\frac{1}{2} \int d x d y a_{\varphi}^{+}(x) M_{\alpha}(x, y) a_{\varphi}^{+}(y)\right) f_{0}^{0},
$$

где $c_{\alpha}=(\operatorname{det}(1+\alpha A))^{-1 / 2}, M_{\alpha}$-ядро оператора $-\alpha A(1+\alpha A)^{-1}$. Пусть $f_{\alpha}^{s}, s=1,2, \ldots$, - решение задачи Коши

$$
\frac{d f_{\alpha}^{s}}{d \alpha}=-B_{0} f_{\alpha}^{s}-\sum_{\substack{i \geqslant 1 \\ s-i \geqslant 0}} B_{i} f_{\alpha}^{s-i}, \quad f_{\alpha}^{0}=0 .
$$

Положим

$$
\begin{aligned}
& \rho_{N}^{(m)}\left(p_{1}, q_{1}, \ldots, p_{N}, q_{N}\right)=(2 \pi m \theta)^{-N d / 2} \exp \left(-\sum_{i=1}^{N} \frac{p_{i}^{2}}{2 m \theta}\right) \\
& \quad \times\left(K_{\varphi, N}\left(f_{1}^{0}+\frac{1}{N^{1 / 2}} f_{1}^{1}+\cdots+\frac{1}{N^{(m-1) / 2}} f_{1}^{m-1}\right)\left(q_{1}, \ldots, q_{N}\right)\right)^{2} .
\end{aligned}
$$


ТеОрема 2. При $\left|g_{1} / \theta\right|<C_{2}, \ldots,\left|g_{p} / \theta\right|<C_{2}$ справедливо соотношение

$$
\int d p_{1} \cdots d p_{N} d q_{1} \cdots d q_{N}\left|\frac{1}{Z_{\exp }} \exp \left(-\frac{H_{N}}{\theta}\right)-\rho_{N}^{(m)}\right|=O\left(N^{-m / 2}\right) .
$$

Решение системы рекуррентных соотношений (14) строится ниже.

ЗАмечАние 1 . При $m=1 \rho_{N}^{(1)}=\rho_{N}^{\text {as. }}$. Таким образом, утверждение 2) теоремы 1 является следствием теоремы 2.

ЗАмЕчАнИЕ 2. Утверждение 1) теоремы 1 следует из утверждения 2). Действительно, согласно [5], [8]

$$
\left\|K_{\varphi, N} f_{1}^{0}\right\|_{L^{2}\left(\mathscr{M}^{N}\right)}^{2} \rightarrow\left\|f_{1}^{0}\right\|, \quad N \rightarrow \infty .
$$

Эта величина в соответствии с [7] равна $\left(\operatorname{det}\left(1-M^{2}\right)\right)^{-1 / 4}$. Поэтому

$$
\lim _{N \rightarrow \infty} \int d p_{1} d q_{1} \cdots d p_{N} d q_{N} \rho_{N}^{\text {as }}\left(p_{1}, q_{1}, \ldots, p_{N}, q_{N}\right)=(\operatorname{det}(1+2 A))^{-1 / 2} .
$$

\section{3. Существование и единственность экстремума свободной энергии.}

ДокАЗАТЕЛЬСтво лЕммы 1 . Приравнивание к 0 вариации $\delta F$ при условии (6) дает уравнение

$$
\begin{aligned}
& g_{1} U_{1}(x)+2 g_{2} \int U_{2}\left(x, y_{1}\right) n_{0}\left(y_{1}\right) d y_{1}+\cdots \\
& \quad+p g_{p} \int U_{p}\left(x, y_{1}, \ldots, y_{p-1}\right) n_{0}\left(y_{1}\right) \cdots n_{0}\left(y_{p-1}\right)+\theta \ln n_{0}(x)=\lambda
\end{aligned}
$$

где $\lambda=$ const. Докажем, что уравнение (16) имеет единственное решение при условии (6). Подстановкой

$$
n_{0}(x)=\frac{e^{-W(x) / \theta}}{\int d x e^{-W(x) / \theta}}
$$

уравнение (16) приводится к виду

$$
W=F[W]
$$

где отображение $F$ :

$$
\begin{aligned}
F[W](x)= & \sum_{l=1}^{p} p g_{p} \int U_{p}\left(x, y_{1}, \ldots, y_{p-1}\right) \exp \left(-\frac{W\left(y_{1}\right)+\cdots+W\left(y_{p-1}\right)}{\theta}\right) \\
& \times d y_{1} \cdots d y_{p-1} / \int \exp \left(-\frac{W\left(y_{1}\right)+\cdots+W\left(y_{p-1}\right)}{\theta}\right) d y_{1} \cdots d y_{p-1} .
\end{aligned}
$$

Докажем, что отображение $F$ является сжимающим по норме $\sup _{x}|W(x)|$. Положим

$$
w(y)=W(y)+\theta \ln \int d y e^{-W(y) / \theta} .
$$

Так как для положительных функций $\varphi_{1}, \varphi_{2}$ справедливо свойство

$$
\min \frac{\varphi_{1}}{\varphi_{2}} \leqslant \frac{\int d y \varphi_{1}(y)}{\int d y \varphi_{2}(y)} \leqslant \max \frac{\varphi_{1}}{\varphi_{2}}
$$


имеет место

$$
\left|\theta \ln \frac{\int d y e^{-W_{1}(y) / \theta}}{\int d y e^{-W_{2}(y) / \theta}}\right| \leqslant\left\|W_{1}-W_{2}\right\| .
$$

Отсюда вытекает, что $\left\|w_{1}-w_{2}\right\| \leqslant 2\left\|W_{1}-W_{2}\right\|$.

В силу соотношения $\left|e^{a}-e^{b}\right| \leqslant\left(e^{a}+e^{b}\right)|a-b|$ имеем

$$
\begin{aligned}
& \left|F\left[W_{1}\right](x)-F\left[W_{2}\right](x)\right| \leqslant \sum_{l=1}^{p} l g_{l} \int d y_{1} \cdots d y_{l-1} U_{l}\left(x, y_{1}, \ldots, y_{l-1}\right) \\
& \quad \times\left|\exp \left(-\frac{w_{1}\left(y_{1}\right)+\cdots+w_{1}\left(y_{l}\right)}{\theta}\right)-\exp \left(-\frac{w_{2}\left(y_{1}\right)+\cdots+w_{2}\left(y_{l}\right)}{\theta}\right)\right| \\
& \quad \leqslant \sum_{l=1}^{p} \max U_{l} 2 l(l-1) g_{l} \frac{\max \left|w_{1}-w_{2}\right|}{\theta} \leqslant \sum_{l=1}^{p} \frac{4 l(l-1) g_{l}}{\theta} \max U_{p}\left\|W_{1}-W_{2}\right\| .
\end{aligned}
$$

Таким образом, при малых $g_{1} / \theta, \ldots, g_{p} / \theta$ отображение $F$ является сжимаюшим, а значит, существует единственное решение уравнения (17) в соответствии с принципом сжимающих отображений (см., например, [9]).

4. Тождественное преобразование канонического распределения. Представим каноническое распределение через одночастичную плотность $n_{0}$ и функции $V_{s}$ (8). Рассмотрим преобразование $S_{l}$, отображающее совокупность функций $\left(u_{0}, \ldots, u_{p}\right)$, $u_{k}: \mathscr{M}^{k} \rightarrow \mathbb{R}$, в совокупность функций $\left(\widetilde{u}_{0}, \ldots, \widetilde{u}_{p}\right)$ вида

$$
\begin{gathered}
\widetilde{u}_{p}=u_{p}, \ldots, \widetilde{u}_{l+1}=u_{l+1}, \\
\widetilde{u}_{l}\left(y_{1}, \ldots, y_{l}\right)=\sum_{s=0}^{l}(-1)^{s} \int d \xi_{1} \cdots d \xi_{s} n_{0}\left(\xi_{1}\right) \cdots n_{0}\left(\xi_{s}\right) \\
\times \sum_{1 \leqslant i_{1}<\cdots<i_{s} \leqslant N} u_{l}\left(\xi_{1}, \ldots, \xi_{s}, y_{1}, \ldots, y_{i_{1}-1}, y_{i_{1}+1}, \ldots, y_{i_{s}-1}, y_{i_{s}+1}, \ldots, y_{l}\right), \\
\widetilde{u}_{l-s}\left(y_{1}, \ldots, y_{l-s}\right)=u_{l-s}\left(y_{1}, \ldots, y_{l-s}\right)-(-1)^{s} \frac{l !}{s !(l-s) !} \int d \xi_{1} \cdots d \xi_{s} n_{0}\left(\xi_{1}\right) \cdots n_{0}\left(\xi_{s}\right) \\
\quad \times u_{l}\left(\xi_{1}, \ldots, \xi_{s}, y_{1}, \ldots, y_{l-s}\right), \quad s \leqslant l .
\end{gathered}
$$

Непосредственно из приведенных формул вытекают следующие свойства преобразования $S_{l}$.

ЛЕмма 2. Выполнены соотношения:

$$
\begin{gathered}
\int d y_{1} n_{0}\left(y_{1}\right) \widetilde{u}_{l}\left(y_{1}, \ldots, y_{l}\right)=0 \\
\sum_{k=0}^{p} \int d x_{1} \cdots d x_{k} n\left(x_{1}\right) \cdots n\left(x_{k}\right)\left(u_{k}\left(x_{1}, \ldots, x_{k}\right)-\widetilde{u}_{k}\left(x_{1}, \ldots, x_{k}\right)\right)=0, \\
\int_{\mathscr{M}} n(x) d x=1, \\
\sum_{k=0}^{p} N^{1-k} \sum_{i_{1}, \ldots, i_{k}=1}^{N} u_{k}\left(x_{i_{1}}, \ldots, x_{i_{k}}\right)=\sum_{k=0}^{p} N^{1-k} \sum_{i_{1}, \ldots, i_{k}=1}^{N} \widetilde{u}_{k}\left(x_{i_{1}}, \ldots, x_{i_{k}}\right) .
\end{gathered}
$$


Отметим, что соотношение (19) является непосредственньг следствием (18), если положить

$$
n(x)=\frac{1}{N}\left(\delta\left(x-x_{1}\right)+\cdots+\delta\left(x-x_{N}\right)\right) .
$$

Рассмотрим композищию преобразований $S_{l}$,

$$
S=S_{2} \cdots S_{p}:\left(0, \frac{g_{1} U_{1}}{\theta}, \ldots, \frac{g_{p} U_{p}}{\theta}\right) \mapsto\left(V_{0}, \ldots, V_{p}\right) .
$$

Докажем, что $V_{2}, \ldots, V_{p}$ имеют вид (8). В силу (18)

$$
\frac{1}{\theta} E[n(\cdot)]=\sum_{l=0}^{p} \int V_{l}\left(x_{1}, \ldots, x_{l}\right) n\left(x_{1}\right) \cdots n\left(x_{l}\right) d x_{1} \cdots d x_{l} .
$$

Приравнивая $s$-ю вариацию левой и правой части формулы (20) при условии (6) и пользуясь свойством (9), получаем формулу (8).

Лемма 3. Выполнено соотношение $V_{1}(x)=-\ln n_{0}(x)+A$, əде $A=$ const.

Доказательство заключается в подстановке

$$
n(x)=n_{0}(x)+\frac{\partial}{\partial x} \alpha(x)
$$

в формулу (20) и приравнивании к 0 слагаемых линейных по $\alpha(x)$.

Лемма 4. Справедливо тождество

$$
\begin{aligned}
e^{-H_{N} / \theta}= & (2 \pi m \theta)^{-N d / 2} \exp \left(-\sum_{i=1}^{N} \frac{p_{i}^{2}}{2 m \theta}\right) C_{N} n_{0}\left(q_{1}\right) \cdots n_{0}\left(q_{N}\right) \\
& \times \exp \left(-\frac{1}{N} \sum_{i_{1}, i_{2}} V_{2}\left(q_{i_{1}}, q_{i_{2}}\right)-\cdots-\frac{1}{N^{p-1}} \sum_{i_{1}, \ldots, i_{p}} V_{p}\left(q_{i_{1}}, \ldots, q_{i_{p}}\right)\right),
\end{aligned}
$$

əде $C_{N}=Z_{\text {exp }}$.

ДокАЗАТЕЛЬСТво. Из леммы 3 вытекает формула $(21) п р и C_{N}=e^{-N\left(V_{0}+A\right)-N f_{0} / \theta}$. Для проверки соотношения $C_{N}=Z_{\text {exp }}$ достаточно установить, что

$$
V_{0}+A=\frac{1}{\theta} F\left[n_{0}(\cdot)\right]
$$

Подставляя в формулу (20) $n=n_{0}$ и пользуясь свойством (9) и леммой 3 , получаем, что

$$
\frac{1}{\theta} E\left[n_{0}(\cdot)\right]=V_{0}+A-\int d x n_{0}(x) \ln n_{0}(x),
$$

а это в силу (7) равносильно формуле (22). Лемма 4 доказана.

5. Построение формального асимптотического ряда. Для доказательства асимптотической формулы (15) удобно ввести понятие полуплотности [2]. Определим полуплотность $\varphi_{N}\left(q_{1}, \ldots, q_{N}\right)$ из соотношения

$$
\frac{1}{Z_{\exp }} e^{-H_{N} / \theta}=(2 \pi m \theta)^{-N d / 2} \exp \left(-\sum_{i=1}^{N} \frac{p_{i}^{2}}{2 m \theta}\right)\left(\varphi_{N}\left(q_{1}, \ldots, q_{N}\right)\right)^{2} .
$$


ЛЕмма 5. Для того чтобы выполнялась формула (15), необходимо и достаточно, чтобы

$$
\left\|\varphi_{N}-K_{\varphi, N}\left(f_{0}+\frac{1}{N^{1 / 2}} f_{1}+\cdots+\frac{1}{N^{(m-1) / 2}} f_{m-1}\right)\right\|_{L^{2}\left(\mathscr{M}^{N}\right)}=O\left(N^{m / 2}\right) .
$$

Доказательство этой леммы приведено в [2].

Построим формальньй асимптотический ряд для полуплотности. Оценка невязки приводится в следующем пункте.

Согласно лемме 4 функция $\varphi_{N}$ имеет вид

$$
\varphi_{N}\left(q_{1}, \ldots, q_{N}\right)=\varphi\left(q_{1}\right) \cdots \varphi\left(q_{N}\right) \exp \left(-R\left(q_{1}, \ldots, q_{N}\right)\right),
$$

где

$$
R=\frac{1}{2 N} \sum_{i_{1}, i_{2}} V_{2}\left(q_{i_{1}}, q_{i_{2}}\right)+\cdots+\frac{1}{2 N^{p-1}} \sum_{i_{1}, \ldots, i_{p}} V_{p}\left(q_{i_{1}}, \ldots, q_{i_{p}}\right) .
$$

Положим

$$
\varphi_{N}^{\alpha}\left(q_{1}, \ldots, q_{N}\right)=\exp \left(-\alpha R\left(q_{1}, \ldots, q_{N}\right)\right) \varphi\left(q_{1}\right) \cdots \varphi\left(q_{N}\right),
$$

где $\alpha \in[0,1]$. Функция (24) удовлетворяет уравнению

$$
\frac{\partial}{\partial \alpha} \varphi_{N}^{\alpha}=-R \varphi_{N}^{\alpha}
$$

и начальному условию $\varphi_{N}^{0}=K_{\varphi, N} \Phi_{0}$, где $\Phi_{0}=(1,0,0, \ldots)$ - вакуумньй вектор пространства Фока.

Приближенное решение уравнения (25) строится по методу работы [8]. Из результатов [8] вытекает лемма.

Лемма 6. Выполнено соотношение $R K_{\varphi, N}=K_{\varphi, N} B$, где $B$ имеет вид (12).

Асимптотическое решение уравнения (25) с точностью $O\left(N^{-m / 2}\right)$ имеет вид

$$
\varphi_{N}^{\alpha}=K_{\varphi, N}\left(f_{\alpha}^{0}+\frac{1}{N^{1 / 2}} f_{\alpha}^{1}+\cdots+\frac{1}{N^{(m-1) / 2}} f_{\alpha}^{m}\right)
$$

где

$$
\left(\frac{\partial}{\partial \alpha}+B\right)\left(f_{\alpha}^{0}+\cdots+\frac{1}{N^{(m-1) / 2}} f_{\alpha}^{m}\right)=O\left(N^{-m / 2}\right),
$$

$f_{i}^{0}=0$ при $i>0 ; f_{0}^{0}=\Phi_{0}$.

Соотношение (26) равносильно соотношениям (13), (14). Отметим, что выражение (13) имеет смысл, если [7]

$$
\left\|\alpha A(1+\alpha A)^{-1}\right\|<1, \quad \alpha \in[0,1] .
$$

При достаточно малых $g_{1} / \theta, \ldots, g_{p} / \theta$ данное соотношение выполнено. Установим важное для дальнейшего свойство функции $M_{\alpha}(x, y)$.

ЛЕмма 7. При достаточно малых $g_{1} / \theta, \ldots, g_{p} / \theta$ выполнено соотношение

$$
\sup \frac{\left|M_{\alpha}(x, y)\right|}{\varphi(x) \varphi(y)}<\frac{1}{8}, \quad \alpha \in[0,1]
$$


ДокАЗАТЕльство. Из формулы (10) для оператора $A$ следует, что ядро оператора

$$
M_{\alpha}=-\alpha A+\alpha^{2} A^{2}-\alpha^{3} A^{3}+\cdots
$$

удовлетворяет свойству

$$
\sup \frac{\left|M_{\alpha}(x, y)\right|}{\varphi(x) \varphi(y)} \leqslant \sum_{n=1}^{\infty} \alpha^{n}\left(\max \left|V_{2}\right|\right)^{n}=\frac{\alpha \max \left|V_{2}\right|}{1-\alpha \max \left|V_{2}\right|} .
$$

При достаточно малых $g_{1} / \theta, \ldots, g_{p} / \theta \max V_{2} \leqslant 1 / 9$, отсюда получаем утверждение леммы.

Построим решение задачи Коши (14). Для краткости введем обозначение

$$
\frac{1}{2} a^{+} M_{\alpha} a^{+} \equiv \frac{1}{2} \int a_{\varphi}(x) M_{\alpha}(x, y) a_{\varphi}^{+}(y) d x d y .
$$

Уравнение (14) на $f_{\alpha}^{s}$ приводится к виду

$$
\frac{\partial f_{\alpha}^{s}}{\partial \alpha}=-\frac{1}{2}\left(a^{+}+a^{-}\right) A\left(a^{+}+a^{-}\right) f_{\alpha}^{s}+g_{\alpha}^{s},
$$

где $g_{\alpha}^{s} \in \mathscr{F}_{\varphi}$ и полином $Q_{\alpha}^{s}$ по операторам $a_{\varphi}^{+}$таковы, что

$$
\begin{gathered}
g_{\alpha}^{s}=Q_{\alpha}^{s}\left(a^{+}\right) e^{\left(a^{+} M_{\alpha} a^{+}\right) / 2} \Phi_{0} \\
Q_{\alpha}^{s}\left(a^{+}\right)=\sum_{l} \int Q_{\alpha, l}^{s}\left(y_{1}, \ldots, y_{l}\right) a_{\varphi}^{+}\left(y_{1}\right) \cdots a_{\varphi}^{+}\left(y_{l}\right) d y_{1} \cdots d y_{l} .
\end{gathered}
$$

Решение уравнения (27) имеет вид

$$
f_{\alpha}^{s}=(\operatorname{det}(1+\alpha A))^{-1 / 2} \mathscr{P}_{\alpha}^{s}\left(a^{+}\right) e^{\left(a^{+} M_{\alpha} a^{+}\right) / 2} \Phi_{0},
$$

где $\mathscr{P}_{\alpha}^{s}-$ полином по $a_{\varphi}^{+}$. Коэффициентные функции удовлетворяют уравнению

$$
\frac{\partial \mathscr{P}_{\alpha, l}^{s}\left(y_{1}, \ldots, y_{l}\right)}{\partial \alpha}=R_{\alpha, l}^{s}\left(y_{1}, \ldots, y_{l}\right)-\sum_{i=1}^{l} \int L\left(y_{i}, z_{i}\right) \mathscr{P}_{\alpha, l}^{s}\left(y_{1}, \ldots, y_{i-1}, z_{i}, y_{i+1}, \ldots, z_{l}\right) .
$$

Здесь

$$
R_{\alpha, l}^{s}\left(y_{1}, \ldots, y_{l}\right)=Q_{\alpha, l}^{s}\left(y_{1}, \ldots, y_{l}\right)-\frac{(l+1)(l+2)}{2} \int A\left(\xi_{1}, \xi_{2}\right) \mathscr{P}_{\alpha, l+2}^{s}\left(\xi_{1}, \xi_{2}, y_{1}, \ldots, y_{l}\right)
$$

a $L$ - ядро оператора $L=(1+\alpha A)^{-1} A$. Если $Q_{\alpha, l}^{s}-$ полином степени $l_{0}$, то положим $\mathscr{P}_{\alpha, l}^{s}=0$ при $l>l_{0}$. Построим решение системы (28). Пусть $\Lambda(\xi, \eta)$ - ядро оператора $\Lambda_{\alpha}=1+\alpha A$. Тогда функции $\mathscr{P}_{\alpha, l}^{s}$ имеют вид

$$
\begin{aligned}
\mathscr{P}_{\alpha, l}^{s}\left(y_{1}, \ldots, y_{l}\right)= & \int d z_{1} \cdots d z_{l} \Lambda_{\alpha}^{-1}\left(y_{1}, z_{1}\right) \cdots \Lambda_{\alpha}^{-1}\left(y_{l}, z_{l}\right) \\
& \times \int_{0}^{\alpha} d \beta \Lambda_{\beta}\left(z_{1}, \zeta_{1}\right) \cdots \Lambda_{\beta}\left(z_{l}, \zeta_{l}\right) R_{\beta, l}^{s}\left(\zeta_{1}, \ldots, \zeta_{l}\right) .
\end{aligned}
$$

Из формулы (29) следует, что коэффициентные функции $\mathscr{P}_{\alpha, l}^{s}$ ограничены, если ограничены функции $Q_{\alpha, l}^{s}$. Ограниченность функций $Q_{\alpha, l}^{s}$ следует, в свою очередь, из ограниченности функций $\mathscr{P}_{\alpha, l}^{s-1}$ и определения (12) оператора $B$. Таким образом, по индукции доказана лемма. 
ЛЕмма 8. Величина

$$
\zeta_{\alpha, m}^{N}=N^{m / 2}\left(\frac{\partial}{\partial \alpha}+B\right)\left(f_{\alpha}^{0}+\cdots+\frac{1}{N^{(m-1) / 2}} f_{\alpha}^{m-1}\right)
$$

имеет вид

$$
\sum_{k=0}^{k_{0}} N^{-k / 2} T_{\alpha}^{k}\left(a^{+}\right) e^{\left(a^{+} M_{\alpha} a^{+}\right) / 2} \Phi_{0}
$$

причем коэффициентные функиии полиномов $T_{\alpha}^{k}$ от операторов $a_{\varphi}^{+}$являются ограниченными.

6. Оценка невязки. Используя леммы 7 и 8 , оценим невязку

$$
\delta_{N, m}^{\alpha}=\varphi_{N}^{\alpha}-K_{\varphi, N} \sum_{s=0}^{m-1} f_{\alpha}^{s} N^{-s / 2}
$$

удовлетворяющую соотношению

$$
\left(\frac{\partial}{\partial \alpha}+R\right) \delta_{N, m}^{\alpha}=-N^{-m / 2} K_{\varphi, N} \zeta_{\alpha, N}^{m}
$$

и начальному условию $\delta_{N, m}^{0}=0$. Имеем

$$
\delta_{N, m}^{\alpha}=-\int_{0}^{1} d \alpha e^{-(1-\alpha) R} K_{\varphi, N} \zeta_{\alpha, N}^{m} N^{-m / 2} .
$$

Норма невязки не превосходит

$$
\delta_{N, m}^{\alpha} \leqslant \sup _{\alpha}\left\|e^{-(1-\alpha) R} K_{\varphi, N} \zeta_{\alpha, N}^{m}\right\|_{L^{2}\left(\mathscr{M}^{N}\right)} N^{-m / 2} .
$$

Таким образом, для проверки формулы (23), а значит, для доказательства теорем 1 и 2 , достаточно доказать лемму.

\section{ЛЕмма 9. Интеграл}

$$
I_{\alpha, N}^{m}=\int d x_{1} \cdots d x_{N} e^{-2(1-\alpha) R\left(x_{1}, \ldots, x_{N}\right)}\left(K_{\varphi, N} \zeta_{\alpha, N}^{m}\right)^{2}\left(x_{1}, \ldots, x_{N}\right)
$$

ограничен равномерно по $\alpha$ и где

ДокАЗАтЕльство. В силу неравенства Коши-Буняковского $I_{\alpha, N}^{m} \leqslant \sqrt{A_{\alpha, N}^{m} B_{\alpha, N}^{m}}$,

$$
\begin{gathered}
A_{\alpha, N}^{m}=\int d x_{1} \cdots d x_{N} e^{-4(1-\alpha) R\left(x_{1}, \ldots, x_{N}\right)} \varphi^{2}\left(x_{1}\right) \cdots \varphi^{2}\left(x_{N}\right), \\
B_{\alpha, N}^{m}=\int \frac{d x_{1} \cdots d x_{N}}{\varphi^{2}\left(x_{1}\right) \cdots \varphi^{2}\left(x_{N}\right)}\left(K_{\varphi, N} \zeta_{\alpha, N}^{m}\right)^{4}\left(x_{1}, \ldots, x_{N}\right) .
\end{gathered}
$$

Проверим ограниченность интегралов (30) и (31).

Для оценки интеграла (30) сведем задачу к случаю $L=1, \varphi=1$. Для этого рассмотрим отображение $T^{d} \rightarrow T^{d}$. Положим

$$
n_{s}\left(\xi^{s}, \ldots, \xi^{d}\right)=\int d \eta^{1} \cdots d \eta^{s-1} \rho\left(\eta^{1}, \ldots, \eta^{s-1}, \xi^{s}, \ldots, \xi^{d}\right), \quad s=1, \ldots, d .
$$

Введем замену

$$
y_{s}\left(\xi^{s}, \ldots, \xi^{d}\right)=\frac{1}{n_{s+1}\left(\xi^{s+1}, \ldots, \xi^{d}\right)} \int_{0}^{\xi^{s}} d \eta^{s} n_{s}\left(\eta^{s}, \xi^{s+1}, \ldots, \xi^{d}\right)
$$


Лемма 10. 1) Отображсение (32) действует из $T^{d}$ в $T^{d}$.

2) Якобиан замены (32) равен $D y / D \xi=\varphi^{2}$.

Обозначим через $W_{l}$ функцию вида $W_{l}\left(y_{1}\left(\xi_{1}\right), \ldots, y_{l}\left(\xi_{l}\right)\right)=V_{l}\left(\xi_{1}, \ldots, \xi_{l}\right)$. Интеграл (30) принимает вид

$$
\int d y_{1} \cdots d y_{N} \exp \left(-\frac{1}{N} \sum_{i_{1}, i_{2}} W_{2}\left(y_{i_{1}}, y_{i_{2}}\right)-\cdots-\frac{1}{N^{p-1}} \sum_{i_{1}, \ldots, i_{p}} W_{p}\left(y_{i_{1}}, \ldots, y_{i_{p}}\right)\right) .
$$

Рассмотрим ортонормированный базис в пространстве $L^{2}\left(T^{d}\right)$

$$
f_{\left(n_{1} \ldots n_{d}\right)}\left(\xi^{1}, \ldots, \xi^{d}\right)=g_{n_{1}}\left(\xi^{1}\right) \cdots g_{n_{d}}\left(\xi^{d}\right),
$$

где $g_{0}(\xi)=1, g_{2 n-1}(\xi)=\sqrt{2} \sin (2 \pi n \xi), g_{2 n}(\xi)=\sqrt{2} \cos (2 \pi n \xi)$. Пронумеруем элементы базиса (34): $f_{0}, f_{1}, \ldots$, причем $f_{0}=1$. Разложим функции $W_{2}, \ldots, W_{p}$ по этому базису:

$$
W_{l}\left(y_{1}, \ldots, y_{l}\right)=\sum_{j_{1}, \ldots, j_{l}=1}^{\infty} W_{l, j_{1} \ldots j_{l}} f_{j_{1}}\left(y_{1}\right) \cdots f_{j_{l}}\left(y_{l}\right) .
$$

В силу условия (9) коэффициенты вида $W_{l, 0 j_{2} \ldots j_{l}}$ обращаются в 0. Оценим сверху показатель экспоненты в формуле (33). Положим

$$
\alpha_{n}=\frac{1}{N} \sum_{i=1}^{n} f_{n}\left(y_{i}\right) .
$$

В силу выбора базиса $|\alpha|<2^{d / 2}$. Имеем

$$
\begin{aligned}
& \left|\frac{1}{N^{l}} \sum_{i_{1}, \ldots, i_{l}=1}^{N} W_{l}\left(x_{i_{1}}, \ldots, x_{i_{l}}\right)\right|=\left|\sum_{j_{1}, \ldots, j_{l}} W_{l, j_{1} \ldots j_{l}} \alpha_{j_{1}} \cdots \alpha_{j_{l}}\right| \\
& \quad \leqslant \sum_{j_{1}, \ldots, j_{l}}\left|W_{l, j_{1} \ldots j_{l}}\right| \cdot\left|\alpha_{j_{1}}\right| \cdot\left|\alpha_{j_{2}}\right| 2^{d(l / 2-1)} \leqslant \sum_{j_{1}, \ldots, j_{l}}\left|W_{l, j_{1} \ldots j_{l}}\right|\left(\alpha_{j_{1}}^{2}+\alpha_{j_{2}}^{2}\right) 2^{d(l / 2-1)} \\
& \quad \leqslant \sum_{n} \sum_{j_{2}, \ldots, j_{l}=1}^{\infty} 2^{l d / 2}\left|W_{l, n j_{2} \ldots j_{l}}\right| \alpha_{n}^{2} .
\end{aligned}
$$

Таким образом, интеграл (33) не превосходит

$$
\int d y_{1} \cdots d y_{N} \exp \left(\frac{1}{N} \sum_{n=1}^{\infty} \sum_{i, j=1}^{N} c_{n} f_{n}\left(y_{i}\right) f_{n}\left(y_{j}\right)\right),
$$

где

$$
c_{n}=\sum_{l=2}^{p} \sum_{j_{1}, \ldots, j_{l-1}=1}^{\infty} 2^{l d / 2}\left|W_{l, n j_{1} \ldots j_{l-1}}\right| .
$$

Интеграл (35) может быть представлен в виде

$$
\int \prod_{n=1}^{\infty}\left(d \beta_{n} \sqrt{\frac{N}{4 \pi}} e^{-N \beta_{n}^{2} / 4}\right)\left(\int\left(\exp \left(\sum_{n=1}^{\infty} \beta_{n} f_{n}(y) \sqrt{c_{n}}\right)-\sum_{n=1}^{\infty} \beta_{n} \sqrt{c_{n}} f_{n}(y)\right) d y\right)^{N} .
$$


Так как функция $\left(\ln \left(e^{u}-u\right)\right) / u^{2}$ ограничена, для некоторого $\mu$ справедлива оценка $e^{u}-u \leqslant e^{\mu u^{2}}$. Отсюда вытекает, что интеграл (36) не превосходит величины

$$
J=\int \prod_{n=1}^{\infty}\left(d \beta_{n} \sqrt{\frac{N}{4 \pi}} e^{-N \beta_{n}^{2} / 4}\right) \exp \left(2^{d} N \mu\left(\sum_{n} c_{n} \beta_{n}\right)^{2}\right)
$$

Рассмотрим замену переменной

$$
\gamma_{k}=\sum_{n=1}^{\infty} \beta_{n} O_{n k}
$$

где $O$ - ортогональная матрица, первый столбец которой пропорционален $\sqrt{c_{n}}: O_{n 1}=$ $\lambda \sqrt{c_{n}}$. После этой замены имеем

$$
J=\int d \gamma_{1} \sqrt{\frac{N}{4 \pi}} e^{-N \gamma_{1}^{2} / 4} \exp \left(2^{d} N \mu \gamma_{1}^{2}\left(\sum c_{n}\right)^{2}\right)=\left(1-2^{d+2} \mu\left(\sum_{n=1}^{\infty} c_{n}\right)^{2}\right)^{-1 / 2}
$$

Таким образом, при

$$
\sum_{l=2}^{p} \sum_{j_{1}, \ldots, j_{l}=1}^{\infty} 2^{l d / 2}\left|W_{l, j_{1} \ldots j_{l}}\right|<\frac{1}{2^{d / 2+1} \sqrt{\mu}}
$$

интеграл (30) ограничен равномерно по $N$.

Оценим сумму в левой части формулы (37):

$$
\begin{aligned}
\sum_{j_{1}, \ldots, j_{l}}\left|W_{l, j_{1} \ldots j_{l}}\right|= & \sum_{n_{1}, \ldots, n_{l d}} 2^{2 l d}\left(1+4 \pi^{2} \sum_{k=1}^{2 d} n_{k}^{2}\right)^{-l d} \int d y_{1} \cdots d y_{l} \\
& \times g_{n_{1}}\left(y_{1}^{1}\right) \cdots g_{n_{d}}\left(y_{1}^{d}\right) \cdots g_{n_{l d-d+1}}\left(y_{l}^{1}\right) \cdots g_{n_{l d}}\left(y_{l}^{d}\right) \\
& \times\left(1-\sum \frac{\partial^{2}}{\partial y_{i}^{a} \partial y_{i}^{a}}\right)^{l d} W_{l}\left(y_{1}, \ldots, y_{l}\right) \\
\leqslant & 4^{l d} \sum_{n_{1}, \ldots, n_{l d}}\left(1+4 \pi^{2} \sum_{k=1}^{2 d} n_{k}^{2}\right)^{-l d} \sup \left((1-\Delta) W_{l}\right) .
\end{aligned}
$$

При достаточно малых $g_{1} / \theta, \ldots, g_{l} / \theta$ величина $(38)$ не превосходит $1 /\left(2^{d(l+1) / 2+1} \sqrt{\mu}\right)$, значит ограниченность интеграла (30) доказана.

Докажем равномерную ограниченность интеграла (31). Заменой (32) общий случай сводится к случаю $\varphi=1$. Оценим интеграл

$$
\int d x_{1} \cdots d x_{N}\left(K_{1, N} f\right)^{4}\left(x_{1}, \ldots, x_{N}\right)=\left\|K_{1, N} f\right\|_{L^{4}}^{4}
$$

Рассмотрим сначала случай, когда элемент $f$ пространства $\mathscr{F}_{1}$ имеет только $n$-ю компоненту, отличную от 0:

$$
f=\frac{1}{\sqrt{n !}} \int d x_{1} \cdots d x_{n} f_{n}\left(x_{1}, \ldots, x_{n}\right) a_{\varphi}^{+}\left(x_{1}\right) \cdots a_{\varphi}^{+}\left(x_{n}\right) \Phi_{0} .
$$


Имеем

$$
\begin{aligned}
\left\|K_{1, N} f\right\|_{L^{4}}^{4}= & \sum_{\substack{i_{1} \neq \cdots \neq i_{n} \\
j_{1} \neq \cdots \neq j_{n}}} \sum_{k_{1} \neq \cdots \neq k_{n}} \sum_{l_{1} \neq \cdots \neq l_{n}} \int d x_{1} \cdots d x_{2 N} \frac{1}{N^{2 n}(n !)^{2}} \\
& \times f_{n}\left(x_{i_{1}}, \ldots, x_{i_{n}}\right) f_{n}\left(x_{j_{1}}, \ldots, x_{j_{n}}\right) f_{n}\left(x_{k_{1}}, \ldots, x_{k_{n}}\right) f_{n}\left(x_{l_{1}}, \ldots, x_{l_{n}}\right) .
\end{aligned}
$$

Заметим, что если хотя бы одно из чисел $1, \ldots, N$ встречается среди индексов

$$
i_{1}, \ldots, i_{n}, \quad j_{1}, \ldots, j_{n}, \quad k_{1}, \ldots, k_{n}, \quad l_{1}, \ldots, l_{n}
$$

только один раз, то в силу соотношения

$$
\int d x_{1} f_{n}\left(x_{1}, \ldots, x_{n}\right)=0
$$

соответствующее слагаемое в сумме (40) обращается в 0. Оценим сверху количество ненулевых слагаемых в формуле (40). Индексы (41) могут пробегать $2 n, 2 n-1, \ldots, n+1$ или $n$ значений. Пусть они пробегают $k$ значений, $n \leqslant k \leqslant 2 n$. Оценим число таких слагаемых: $k$ чисел из набора $1, \ldots, N$ можно выбрать $N ! /((N-k) ! k !)$ способами, индексы $i_{1}, \ldots, i_{n}-k ! / n$ ! способами, столькими же способами можно выбрать индексы $j_{1}, \ldots, j_{n}$; $k_{1}, \ldots, k_{n} ; l_{1}, \ldots, l_{n}$. Таким образом, число ненулевых слагаемых в сумме (40) не превосходит

$$
\Omega \leqslant \sum_{k=n}^{2 n} \frac{N !}{k !(N-k) !}\left(\frac{k !}{n !}\right)^{4} \leqslant(n+1) \frac{N^{2 n}}{(n !)^{4}}((2 n) !)^{3} .
$$

Поэтому

$$
\left\|K_{1, N} f\right\|_{L^{4}}^{4} \leqslant \frac{\left(\sup \left|f_{n}\right|\right)^{4}}{N^{2 n}(n !)^{2}} \Omega \leqslant\left(\sup \left|f_{n}\right|\right)^{4} \frac{((2 n) !)^{3}}{(n !)^{6}}(n+1) .
$$

Пользуясь формулой Стирлинга $n ! \sim(n / e)^{n} \sqrt{2 \pi n}$, получаем, что, начиная с некоторого $n$,

$$
\left\|K_{1, N} f\right\|_{L^{4}} \leqslant \sup \left|f_{n}\right| 8^{n / 2} .
$$

При произвольном $f$ таком, что $\sup \left|f_{k}\right|<\infty$, справедлива оценка

$$
\left\|K_{1, N} f\right\|_{L^{4}} \leqslant c+\sum_{n=0}^{\infty} \sup \left|f_{n}\right| 8^{n / 2}, \quad c=\text { const } .
$$

Таким образом, интеграл (39) сходится, если сходится ряд

$$
\sum_{n=0}^{\infty} \sup \left|f_{n}\right| 8^{n / 2}
$$

Проверим сходимость ряда (42) для вектора $f \in \mathscr{F}_{1}$ вида

$$
f=\int d x_{1} \cdots d x_{k} A\left(x_{1}, \ldots, x_{k}\right) a^{+}\left(x_{1}\right) \cdots a^{+}\left(x_{k}\right) e^{\left(a^{+} M a^{+}\right) / 2} \Phi_{0} .
$$


Отсюда следует равномерная ограниченность интеграла (31). Имеем

$$
f_{k+2 j}\left(x_{1}, \ldots, x_{2 j+k}\right)=\frac{\sqrt{(2 j+k) !}}{2^{j} j !} A\left(x_{2 j+1}, \ldots, x_{2 j+k}\right) M\left(x_{1}, x_{2}\right) \cdots M\left(x_{2 j-1}, x_{2 j}\right) .
$$

Сумма (42) в данном случае не превосходит

$$
\sum_{j=0}^{\infty} \sup |A|(\sup |M|)^{j} \frac{\sqrt{(2 j+k) !}}{2^{j} j !} 8^{k / 2+j} .
$$

При условиях $\sup |M|<1 / 8, \sup |A|<\infty$, проверенных ранее, ряд (42) сходится. Лемма 9 , значит, и теоремы 1 и 2 доказаны.

Автор благодарит академика РАН В.П. Маслова за постановку задачи, постоянное внимание к работе, полезное обсуждение ряда вопросов и ценные замечания.

\section{СПИСОК ЦИТИРОВАННОЙ ЛИТЕРАТУРЫ}

[1] Ландау Л. Д., Лифшиц Е. М. Статистическая физика. Ч. 1. М.: Наука, 1976.

[2] Maslov V.P., Shvedov O. Yu. An asymptotic formula for the $N$-particle density function as $N \rightarrow \infty$ and violation of the chaos hypothesis // Russian J. Math. Phys. 1994. V. 2. № 2. P. 217-234.

[3] Маслов В.П. Комплексный метод ВКБ в нелинейных уравнениях. М.: Наука, 1977.

[4] Кац М. Вероятность и смежные вопросы в физике. М.: Наука, 1965.

[5] Маслов В.П., Шведов О. Ю. Стационарные асимптотические решения задачи многих тел и вывод интегральных уравнений с прыгающей нелинейностью // Дифференц. уравнения. 1995. Т. 31. № 2. С. 312-326.

[6] Маслов В. П. Об интегральном уравнении $u(x)=F(x)+\int G(x, \xi) u_{+}^{k / 2}(\xi) d \xi / \int u_{+}^{k / 2}(\xi) d \xi$ // Функцион. анализ и его прилож. 1994. Т. 28. № 1. С. 41-50.

[7] Березин Ф.А. Метод вторичного квантования. М.: Наука, 1986.

[8] Maslov V.P., Shvedov O. Yu. The chaos conservation problem in quantum physics // Russian J. Math. Phys. 1996. V. 4. № 2. P. 173-216.

[9] Колмогоров А. Н., Фомин С. В. Элементы теории функций и функционального анализа. М.: Наука, 1989.

Московский государственный университет им. М. В. Ломоносова Университет Бристоля (Великобритания) 